\title{
Universality of Returning Electron Wave Packet in High-Order Harmonic Generation with Midinfrared Laser Pulses
}

\author{
Anh-Thu Le, ${ }^{1}$ Hui Wei, ${ }^{1}$ Cheng Jin,,${ }^{1}$ Vu Ngoc Tuoc, ${ }^{2}$ Toru Morishita, ${ }^{3}$ and C. D. Lin ${ }^{1}$ \\ ${ }^{1}$ Department of Physics, Cardwell Hall, Kansas State University, Manhattan, Kansas 66506, USA \\ ${ }^{2}$ Institute of Engineering Physics, Hanoi University of Science and Technology, 1 Dai Co Viet Road, Hanoi 10000, Vietnam \\ ${ }^{3}$ Department of Engineering Science, University of Electro-Communications, 1-5-1 Chofu-ga-oka, Chofu-shi, Tokyo 182-8585, Japan
}

(Received 28 April 2014; published 15 July 2014)

\begin{abstract}
We show that a returning electron wave packet in high-order harmonic generation (HHG) with midinfrared laser pulses converges to a universal limit for a laser wavelength above about $3 \mu \mathrm{m}$. The results are consistent among the different methods: a numerical solution of the time-dependent Schrödinger equation, the strong-field approximation, and the quantum orbits theory. We further analyze how the contribution from different electron "trajectories" survives the macroscopic propagation in the medium. Our result thus provides a new framework for investigating the wavelength scaling law for the HHG yields.
\end{abstract}

DOI: 10.1103/PhysRevLett.113.033001

PACS numbers: 33.80.Eh, 42.65.Ky

High-order harmonic generation (HHG) has been a very active field of research over the last two decades. With the popular Ti:sapphire laser operating at an 800-nm wavelength, a great deal of experimental and theoretical research has been carried out. This interest is due to two facts. First, HHG provides a tabletop coherent extreme ultraviolet (XUV) light source [1] that can be used for application in science and technology. In particular, it can serve as a source of attosecond pulses [2,3] and attosecond-pulse train [4]. Second, the HHG signal contains information about the target structure [5-7]. As few-cycle laser pulses are routinely available, HHG holds great promise to become a spectroscopic tool capable of providing a femtosecond scale temporal resolution.

The typical photon energy range available from $\mathrm{HHG}$ sources with a Ti:sapphire laser has been limited to about $100 \mathrm{eV}$ or so. The well-known cutoff law $\Omega_{c}=I_{p}+$ $3.17 U_{p}$, with $\Omega_{c}, I_{p}$, and $U_{p} \propto I_{0} \lambda^{2}$ being the cutoff energy, the ionization potential of the target, and the ponderomotive energy, respectively, suggests that higher energy photons can be produced with longer driving laser wavelengths $\lambda$ (atomic units are used throughout, unless otherwise indicated). Increasing the peak laser intensity $I_{0}$ is not an option because of the ground-state depletion as well as the phase mismatch caused by excessive free electrons in the medium. With recent development in optical parametric amplification techniques, midinfrared (mid-IR) lasers with a wavelength of a few microns are available today, with a sufficient intensity to generate high harmonics, thus pushing the HHG photon energy range beyond the water window and even to the $\mathrm{keV}$ region [8-11].

Wavelength scaling of HHG yield has been studied both theoretically [12-19] and experimentally [20,21]. Early theoretical investigation was mostly based on numerical solution of the time-dependent Schrödinger equation
(TDSE) [12-14]. These studies showed that, apart from small oscillation on a fine $\lambda$ scale due to the threshold phenomena effect, harmonic yield at constant driving laser intensity drops drastically as $\lambda^{-x}$, with $x \approx 5-6$. All these studies have been limited to wavelengths below $2 \mu \mathrm{m}$, and the analysis mostly for a fixed energy range from 20 to $50 \mathrm{eV}$. More recent studies within the strong-field approximation (SFA) [16-18,22] showed scaling behavior with somewhat slightly different $x$. By using the quantum orbits (QO) theory [23-25], scaling law for long and short trajectories have also been reported $[18,19]$.

At constant laser intensity, Keldysh parameter $\gamma=$ $\sqrt{I_{p} /\left(2 U_{p}\right)}$ scales as $1 / \lambda$. It is therefore expected that longer wavelengths would drive the interaction further into the tunneling regime $(\gamma \ll 1)$, where the semiclassical description (as in the SFA) would be more accurate. For subtleties related to the Keldysh parameter we refer the reader to the recent discussion by Reiss [26] as well as within the framework of the recently developed adiabatic theory [27] for the range of applicability.

According to the quantitative rescattering model [7,28-31], HHG yield is a product of the returning electron wave packet and the photorecombination cross section. The latter is target dependent and varies strongly with energy. As a consequence, the scaling law for HHG yield is not only target specific, but also depends on the HHG photon energy, as evident in Refs. [13,14,31]. The goal of this Letter is to explore the scaling law for the returning electron wave packet. This approach allows us to identify a universal shape of the wave packet as a function of a scaled energy in the long wavelength limit. The universal shape is nearly identical for all atomic targets we considered, including atomic hydrogen, neon, argon, and xenon, for typical laser intensities for each target. The conventional scaling law at fixed range of energy [12-14] can be easily obtained from our universal wave packet. Earlier theoretical 
results [12] showed a quite strong contribution from higherorder returns at the single-atom response level. Here we show that under typical experimental conditions, when good phase matching is achieved in the macroscopic medium, only the first return survives.

Following the quantitative rescattering model, we define the wave packet as

$$
|W(E)|^{2}=S(E) / \sigma(E),
$$

where $S(E)$ is the HHG yield and $\sigma(E)$ is the photorecombination cross section. For each atomic target, the cross section can be exactly calculated numerically within a model potential approach in the single-active electron approximation. The same model potential is used in the TDSE to calculate $S(E)[30,32]$. In the following we will use a scaled energy $\tilde{E}$ defined as $\tilde{E}=\left(E-I_{p}\right) / U_{p}$, which corresponds to the returning electron kinetic energy in units of $U_{p}$. For photon energy much higher than the ionization potential $I_{p}$, quite typical with a mid-IR driving laser pulse, $\tilde{E} \approx E / U_{p}$.

We use three different methods for analysis: the TDSE, the SFA [33], and the QO theory. To be specific, we now focus on the atomic hydrogen target. A laser pulse of twocycle flattop envelope with half-cycle turn-on (turn-off) ramps, as shown in Fig. 1(a), with an intensity of $1 \times 10^{14} \mathrm{~W} / \mathrm{cm}^{2}$, and the wavelengths from 0.8 to $3.2 \mu \mathrm{m}$ is used in the TDSE. For SFA and QO analysis, the wavelength is extended up to $6.4 \mu \mathrm{m}$. Longer laser pulses are not needed since we will mostly focus on the first return.

We show in Fig. 1(b) a typical time-frequency trace of the induced dipole acceleration from the TDSE for the wavelength of $2.4 \mu \mathrm{m}$. This time-frequency trace is calculated using a wavelet transformation [34,35]. We can clearly identify the first return in the time range $t=$ [-0.57: -0.07$]$ in units of optical cycle (OC) [as indicated by the vertical dashed lines in Fig. 1(b)]. The electron, which recombines during this time interval, was born into the continuum at the ionization time near $t_{i} \approx-1$ OC. Note that the first return can be further separated into two branches, associating with the long and short trajectories in the QO theory. For our analysis of the TDSE results in the following, we will define "short"(or "long") trajectory contribution to $\mathrm{HHG}$ as due to the recombination in the time range $t_{r}=[-0.57:-0.3]$ OC $\quad$ or $t_{r}=[-0.3$ : $-0.07]$ OC). The HHG signal due to the first return is the coherent contribution of short and long trajectories. In the subsequent subcycles, higher-order returns start to show up. Since the HHG spectrum and wave packet typically oscillate on a fine energy scale, we smooth out the fast oscillation by using Bezier interpolation. A typical example of the wave packet and its Bezier curve are shown in Fig. 1(c) for the wavelength of $2.4 \mu \mathrm{m}$.
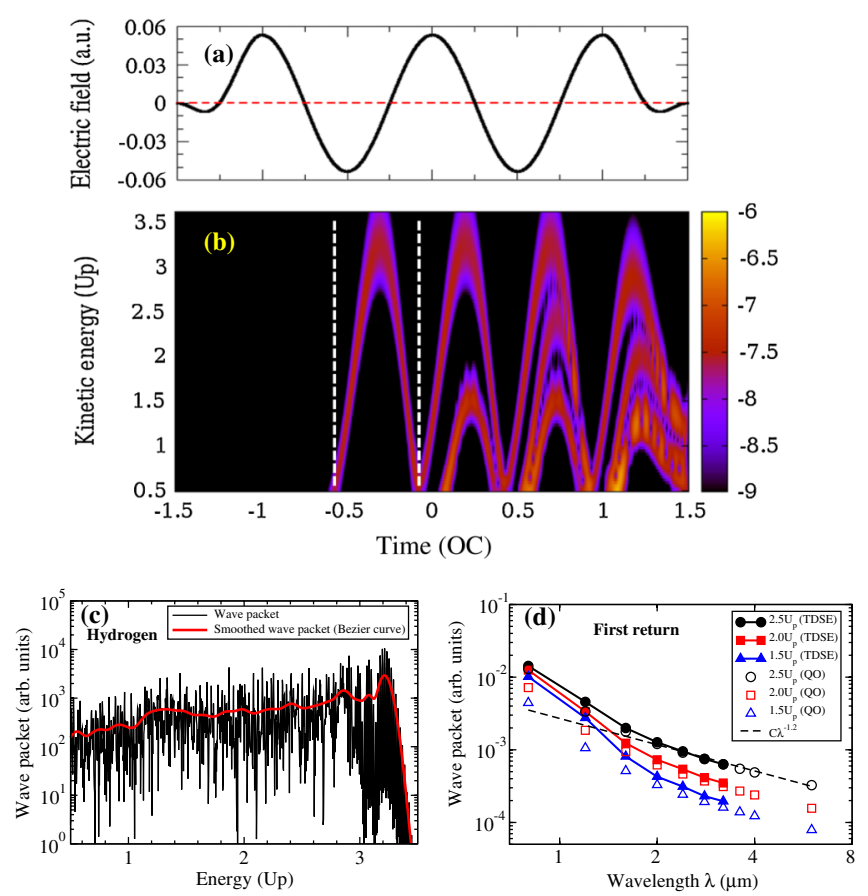

FIG. 1 (color online). (a) Typical flattop electric field used in our calculation. (b) Typical time-frequency trace of the induced dipole acceleration from TDSE. (c) Typical returning electron wave packet and its Bezier curve. (d) TDSE results for electron wave packet $|W(\tilde{E})|^{2}$ due to first return at fixed scaled energies of 2.5, 2.0 , and 1.5 vs laser wavelength from 0.8 to $3.2 \mu \mathrm{m}$. QO results are also shown (symbols), from 0.8 to $6 \mu \mathrm{m}$. All results here are for atomic hydrogen with laser intensity of $1 \times 10^{14} \mathrm{~W} / \mathrm{cm}^{2}$.

In Fig. 1(d) we show the wave packets due to the first return at different scaled energies $\tilde{E}=2.5,2.0$, and 1.5 , as a function of laser wavelength from both TDSE and QO theory. The QO results have been normalized to the TDSE result at $3.2 \mu \mathrm{m}$, for $\tilde{E}=2.5$. It appears that the wave packet scales approximately as $\lambda^{-1.2}$ (dashed line) for both methods at wavelengths above $2.4 \mu \mathrm{m}$ for all three energies considered. At wavelengths shorter than $2.4 \mu \mathrm{m}$, the wave packet drops faster, especially for smaller $\tilde{E}$. This result also indicates that the QO is quite adequate for describing electron wave packet. We note that within the QO the scaling slowly approaches $\lambda^{-1}$ at even longer wavelengths (above $6 \mu \mathrm{m}$ ) [36]. This result also agrees with the numerical result from the SFA. However, at such long wavelengths, one will need to take into account the magnetic-field effect [37]. Therefore, in this Letter we will limit ourselves only to the wavelengths shorter than about $6 \mu \mathrm{m}$, when the scaling is approximately $\lambda^{-1.2}$.

According to the above analysis we now define a rescaled wave packet $|\tilde{W}(\tilde{E})|^{2}=\left(\lambda / \lambda_{0}\right)^{1.2}|W(\tilde{E})|^{2}$, where $\lambda_{0}$ is a reference wavelength, introduced for the dimension purpose. The main result of this Letter is presented in Fig. 2(a) where the rescaled wave packets from the first return are shown as a function of $\tilde{E}$ for different wavelengths at a laser intensity of $1 \times 10^{14} \mathrm{~W} / \mathrm{cm}^{2}$. Just above 

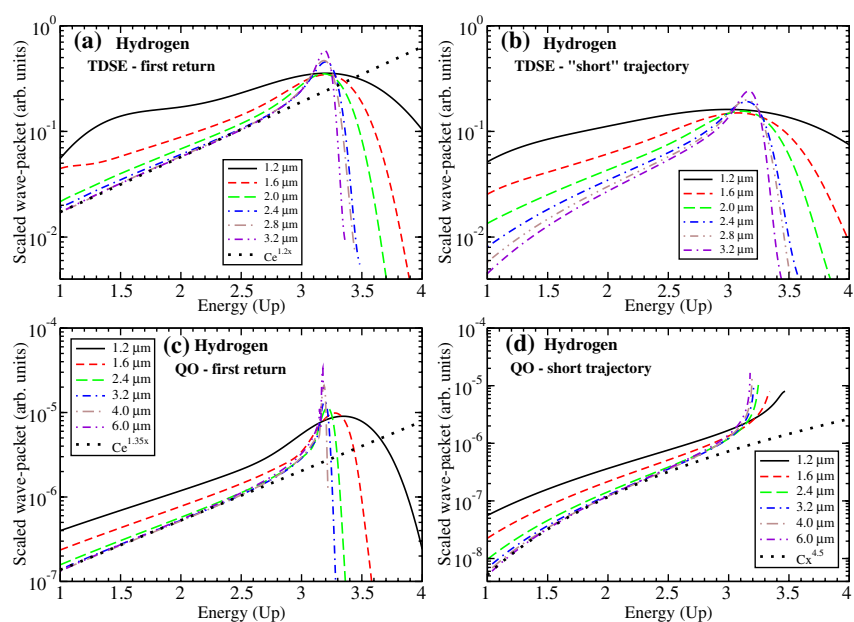

FIG. 2 (color online). (a) TDSE results for rescaled wave packets $|\tilde{W}(\tilde{E})|^{2}$ from atomic hydrogen due to the first return with different laser wavelengths. The curves have been smoothed out using Bezier interpolation. (b) Same as (a), but for short trajectories. (c) and (d) Same as (a) and (b), respectively, but from QO theory.

$0.8 \mu \mathrm{m}$, the rescaled wave packet decreases very fast with increasing wavelength $\lambda$. It then decreases slowly with $\lambda>$ $2.4 \mu \mathrm{m}$ and virtually converges to a common shape for $\tilde{E} \lesssim 3$. This universal shape can be approximated by an exponential function of the form $e^{1.2 \tilde{E}}$ (dotted line). Above $\tilde{E} \approx 3$ the wave packet still experiences small changes with increasing $\lambda$ due to the influence of the abrupt cutoff, which becomes sharper for longer wavelength [12]. Since the short trajectory contributes most to the macroscopic HHG in a typical good phase matching condition, we also show in Fig. 2(b) the rescaled wave packet from the short trajectory vs scaled energy. The convergence appears to be somewhat slower than for the first return. These TDSE results have been confirmed by our QO calculation shown in Fig. 2(c) and 2(d) for the first return and short trajectory, respectively. There are some small differences in the slopes of the wave packets from the two methods. Similarly to the TDSE results, we also notice slower convergence of the short-trajectory wave packet to a universal limit, as compared to the first return.

A nice feature of our approach is that the rescaled wave packet has a universal behavior in the long wavelength limit, nearly independent of the target. As shown in Fig. 3(a), the wave packet due to the first return for different targets with long wavelengths driving the laser are indeed virtually identical. Here laser intensities used in the TDSE calculation are $0.8 I_{0}, 1 I_{0}, 1.5 I_{0}$, and $2 I_{0}$ for $\mathrm{Xe}$, $\mathrm{H}$, Ar, and $\mathrm{Ne}$, respectively, where $I_{0}=10^{14} \mathrm{~W} / \mathrm{cm}^{2}$, while the wavelengths are 3.6, 3.2, 2.8, and $2.4 \mu \mathrm{m}$, respectively. Note that higher laser intensities are used in the calculations for targets with higher $I_{p}$. This intensity range is also quite typical for an efficient phase matching in real experiments [11]. Small modifications in laser intensity
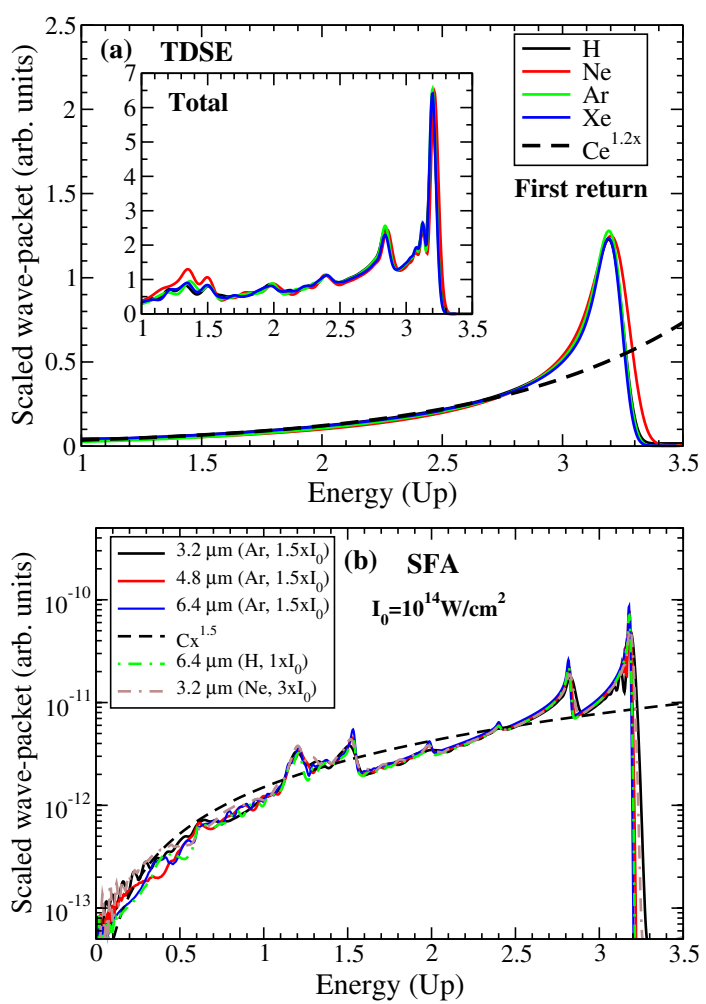

FIG. 3 (color online). (a) Wave packets due to the first return from the TDSE for different atoms as shown in the labels. See text for laser parameters. The same are shown in the inset, but for the total wave packets. (b) Total rescaled wave packets from the SFA for different targets. Laser intensities and wavelengths are given in the labels. In both (a) and (b) the wave packets from different targets have been normalized to account for the differences in ionization rates.

do not change our conclusion. Our choice of the shorter wavelengths used for $\mathrm{Ar}$ and $\mathrm{Ne}$ is mostly for computational convenience as computer time for the TDSE increases drastically with laser wavelength.

The universality of the returning wave packet is not limited to the first return. Indeed, with still relatively short wavelengths used in the TDSE, the total (from the whole laser pulse) rescaled wave packets from different targets are nearly identical; see the inset of Fig. 3(a). We have further carried out calculations for longer wavelengths within the SFA. The SFA results shown in Fig. 3(b) for Ar with different wavelengths confirm the convergence of the total wave packet for $\lambda \gtrsim 3.2 \mu \mathrm{m}$. Furthermore, the total wave packets from $\mathrm{H}$ and $\mathrm{Ne}$ also converge to the same universal shape. Here, all the SFA calculations were done with the pulse shape shown in Fig. 1(a). By using the QO theory we found that not only the first return, but any higher-order return has its own universal wave packet [36] that leads to the universal shape for the total wave packet. We note that a similar study within the SFA on high-order return contributions at the single atom level has been reported recently by $\mathrm{He}$ et al. [38]. 
Overall, the universal total wave packet from a flattop pulse can be approximated by $|\tilde{W}(\tilde{E})|^{2} \propto \tilde{E}^{1.5}$ [dashed line in Fig. 3(b)]. Taking into account that $|\tilde{W}(\tilde{E})|^{2} \propto$ $\lambda^{1.2}|W(\tilde{E})|^{2}, E \approx \tilde{E} U_{p}$, and $U_{p} \propto \lambda^{2}$, it follows that $|W(E)|^{2} \propto \lambda^{-4.2}$ for a fixed $E$. For a fixed energy $E$, the photorecombination cross section only gives an overall factor. The scaling law for HHG yield at a fixed energy $E$ should therefore be the same as for the wave packet $|W(E)|^{2}$. This argument holds approximately for a small range of energy, say, $E=[20-50] \mathrm{eV}$, as used in [12-14]. Their scaling law of $S(E) \propto \lambda^{-(5-6)}$ for HHG yield was somewhat less favorable compared to our result. The difference is mainly due to the fact that earlier analyses [12-14] were limited to wavelengths below $2 \mu \mathrm{m}$, where the wave packet drops faster with wavelength [see Fig. 1(d)]. Our scaling law strictly applies only to the long wavelength limit, typically above $3 \mu \mathrm{m}$, when the wave packet virtually converges to a universal shape.

We emphasize that the universal total wave packet depends on the actual shape (envelope) of the laser pulse. Furthermore, the universal behavior is expected to break down when depletion of the ground state becomes significant within a half optical cycle.

Macroscopic phase matching of HHG has been known to be quite different for short and long trajectories [39]. However, the effect of macroscopic propagation has not been well documented for higher-order returns from midIR pulses. To illustrate this effect, we use the QO theory to separate the different contributions from multiple returns as well as short and long trajectories of the first return. Returns up to the third order are included in our calculation. Our simulations are shown in Fig. 4(a) and 4(b) for a typical gas jet experimental setup with a tightly focused laser beam, for two different gas jet positions at 1 and $3.5 \mathrm{~mm}$ after the focus, respectively. The target is Ar and the laser wavelength is $1.6 \mu \mathrm{m}$. We keep the laser intensity of $1 \times 10^{14} \mathrm{~W} / \mathrm{cm}^{2}$ at the center of the gas jet. The other parameters are the same for the two cases. As evident from Fig. 4(a), long trajectories dominate for the gas jet near the laser focus. Higher-order returns contribute quite insignificantly to the total macroscopic HHG yields, mainly below about $50 \mathrm{eV}$. The contribution comes mostly from the second return which has a cutoff near $I_{p}+1.5 U_{p} \approx 51 \mathrm{eV}$. For a good phase matching case, shown in Fig. 4(b), short trajectories dominate and there is virtually no contribution from higher order returns. This result is not totally surprising since an electron with a higher order return spends a longer amount of time in the continuum, and, therefore, it accumulates a larger phase due to the laser. The variation of laser intensity in the interaction region would wash out a higher order return contribution.

In conclusion, we have shown that a returning electron wave packet in high harmonic generation with mid-IR laser pulses converges to a universal shape at the long wave
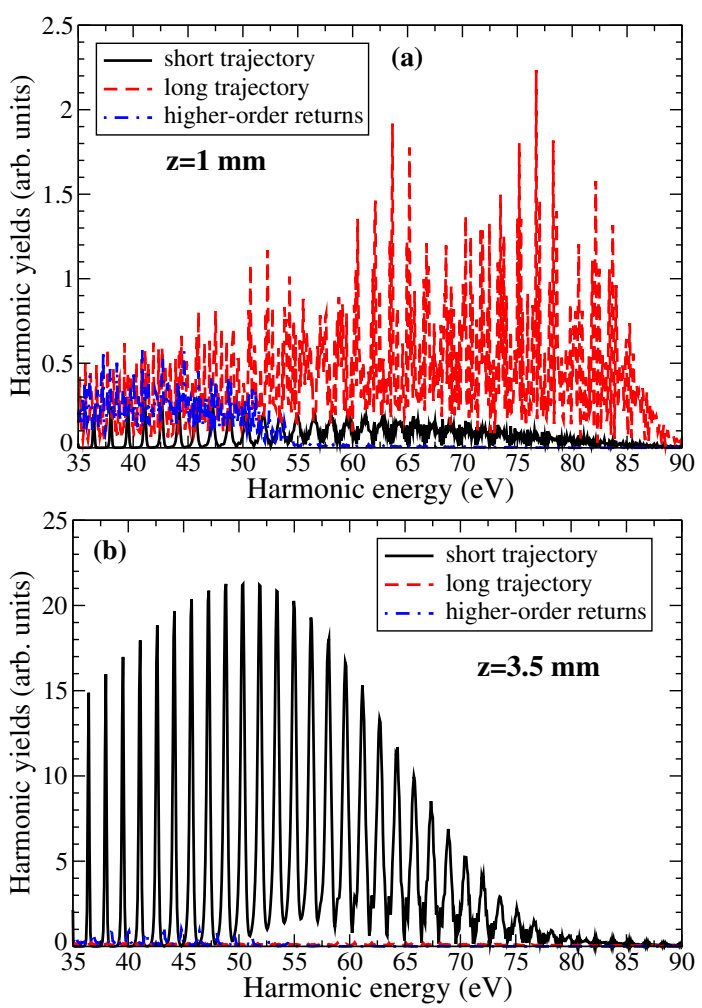

FIG. 4 (color online). Macroscopic HHG yields after propagating in the Ar gas jet of $1 \mathrm{~mm}$ thick placed at $z=1 \mathrm{~mm}$ (a) and $z=3.5 \mathrm{~mm}$ (b) after the laser focus. Single atom induced dipole is calculated by the QO theory, for a sine-squared envelope laser pulse with $1.6 \mu \mathrm{m}$ wavelength, $1 \times 10^{14} \mathrm{~W} / \mathrm{cm}^{2}$ peak intensity (at the center of gas jet), and a 30-cycle total duration. The beam waist is $36 \mu \mathrm{m}$.

length limit. A nearly universal shape starts to occur for wavelengths as short as about $3 \mu \mathrm{m}$ for most atomic targets under typical laser intensities of order $1 \times 10^{14} \mathrm{~W} / \mathrm{cm}^{2}$ or so. This indicates that, with regard to the returning electron wave packet, the main physics at long wavelengths is in fact quite simple and can be explained accurately using semiclassical approaches such as the SFA and the QO theory, even though the numerical calculation of the TDSE is much more demanding, as compared to the case of a Ti: sapphire laser. Thus, the knowledge of the universal rescaled wave packet provides a simple and practical method for quick estimates of HHG yield from different targets with midinfrared laser pulses. We have also shown that higher order returns are quite negligible in good phase matching condition for typical experimental setups with tightly focused laser beams.

This work was supported in part by the Chemical Sciences, Geosciences, and Biosciences Division, Office of Basic Energy Sciences, Office of Science, U.S. Department of Energy. Work by V.N.T. is supported by the Vietnamese National Foundation for Science and Technology Development (NAFOSTED) Grant 
No. 103.01-2014.25. T. M. was supported by Grants-in-Aid for Scientific Research (A), (B), and (C) from the Ministry of Education, Culture, Sports, Science, and Technology, Japan.

[1] T. Popmintchev, M. C. Chen, P. Arpin, M. M. Murnane, and H. C. Kapteyn, Nat. Photonics 4, 822 (2010).

[2] E. Goulielmakis, M. Schultze, M. Hofstetter, V. S. Yakovlev, J. Gagnon, M. Uiberacker, A. L. Aquila, E. M. Gullikson, D. T. Attwood, R. Kienberger, F. Krausz, and U. Kleineberg, Science 320, 1614 (2008).

[3] K. Zhao, Q. Zhang, M. Chini, Y. Wu, X. Wang, and Z. Chang, Opt. Lett. 37, 3891 (2012).

[4] R. López-Martens, K. Varjú, P. Johnsson, J. Mauritsson, Y. Mairesse, P. Salières, M. B. Gaarde, K. J. Schafer, A. Persson, S. Svanberg, C. G. Wahlström, and A. L'Huillier, Phys. Rev. Lett. 94, 033001 (2005).

[5] J. Itatani, J. Levesque, D. Zeidler, H. Niikura, H. Pepen, J. C. Kiefer, P. B. Corkum, and D. M. Villeneuve, Nature (London) 432, 867 (2004).

[6] M. Lein, J. Phys. B 40, R135 (2007).

[7] C. D. Lin, A. T. Le, Z. Chen, T. Morishita, and R. R. Lucchese, J. Phys. B 43, 122001 (2010).

[8] P. Colosima, G. Doumy, C. I. Blaga, J. Wheeler, C. Hauri, F. Catoire, J. Tate, R. Chirla, A. M. March, G. G. Paulus, H. G. Muller, P. Agostini, and L. F. DiMauro, Nat. Phys. 4, 386 (2008).

[9] K. Hong, S. Huang, J. Moses, X. Fu, C. Lai, G. Cirmi, A. Sell, E. Granados, P. Keathley, and F. Kärtner, Opt. Express 19, 15538 (2011).

[10] G. Andriukaitis, T. Balciunas, S. Alisauskas, A. Pugzlys, A. Baltuska, T. Popmintchev, M. C. Chen, M. M. Murnane, and H. C. Kapteyn, Opt. Lett. 36, 2755 (2011).

[11] T. Popmintchev et al., Science 336, 1287 (2012).

[12] J. Tate, T. Auguste, H. G. Muller, P. Salières, P. Agostini, and L. F. DiMauro, Phys. Rev. Lett. 98, 013901 (2007).

[13] K. Schiessl, K. L. Ishikawa, E. Persson, and J. Burgdörfer, Phys. Rev. Lett. 99, 253903 (2007).

[14] M. V. Frolov, N. L. Manakov, and A. F. Starace, Phys. Rev. Lett. 100, 173001 (2008).

[15] V. Yakovlev, M. Ivanov, and F. Krausz, Opt. Express 15, 15351 (2007).

[16] E. L. Falcão-Filho, V. M. Gkortsas, A. Gordon, and F. X. Kätner, Opt. Express 17, 11217 (2009).

[17] J. A. Pérez-Hernández, L. Roso, and L. Plaja, Opt. Express 17, 9891 (2009).
[18] D. R. Austin and J. Biegert, Phys. Rev. A 86, 023813 (2012).

[19] T. Auguste, F. Catoire, P. Agostini, L. F. DiMauro, C. C. Chirila, V. S. Yakovlev, and P. Salières, New J. Phys. 14, 103014 (2012).

[20] B. Shan and Z. Chang, Phys. Rev. A 65, 011804(R) (2001).

[21] A. D. Shiner, C. Trallero-Herrero, N. Kajumba, H.-C. Bandulet, D. Comtois, F. Légaré, M. Giguère, J-C. Kieffer, P. B. Corkum, and D. M. Villeneuve, Phys. Rev. Lett. 103, 073902 (2009).

[22] C. Jin, A. T. Le, and C. D. Lin, Phys. Rev. A 83, 023411 (2011).

[23] P. Salieres, B. Carre, L. Le Deroff, F. Grasbon, G. G. Paulus, H. Walther, R. Kopold, W. Becker, D. B. Milosevic, A. Sanpera, and M. Lewenstein, Science 292, 902 (2001).

[24] D. B. Milosevic and W. Becker, Phys. Rev. A 66, 063417 (2002).

[25] G. Sansone, C. Vozzi, S. Stagira, and M. Nisoli, Phys. Rev. A 70, 013411 (2004).

[26] H. R. Reiss, Phys. Rev. A 82, 023418 (2010).

[27] O. I. Tolstikhin and T. Morishita, Phys. Rev. A 86, 043417 (2012).

[28] T. Morishita, A. T. Le, Z. Chen, and C. D. Lin, Phys. Rev. Lett. 100, 013903 (2008).

[29] A. T. Le, R. R. Lucchese, M. T. Lee, and C. D. Lin, Phys. Rev. Lett. 102, 203001 (2009).

[30] A. T. Le, R. R. Lucchese, S. Tonzani, T. Morishita, and C. D. Lin, Phys. Rev. A 80, 013401 (2009).

[31] M. V. Frolov, N. L. Manakov, T. S. Sarantseva, M. Yu. Emelin, M. Yu. Ryabikin, and A. F. Starace, Phys. Rev. Lett. 102, 243901 (2009).

[32] T. Morishita, Z. Chen, S. Watanabe, and C. D. Lin, Phys. Rev. A 75, 023407 (2007).

[33] M. Lewenstein, Ph. Balcou, M. Yu. Ivanov, A. L'Huillier, and P. B. Corkum, Phys. Rev. A 49, 2117 (1994).

[34] X. M. Tong and S.-I. Chu, Phys. Rev. A 61, 021802(R) (2000).

[35] P. Antoine, B. Piraux, and A. Maquet, Phys. Rev. A 51, R1750 (1995).

[36] A. T. Le, H. Wei, C. Jin, and C. D. Lin (to be published).

[37] C. Hernandez-Garcia, J. A. Perez-Hernandez, T. Popmintchev, M. M. Murnane, H. C. Kapteyn, A. JaronBecker, A. Becker, and L. Plaja, Phys. Rev. Lett. 111, 033002 (2013).

[38] L. He, Y. Li, Z. Wang, Q. Zhang, P. Lan, and P. Lu, Phys. Rev. A 89, 053417 (2014).

[39] M. B. Gaarde, J. L. Tate, and K. J. Schafer, J. Phys. B 41, 132001 (2008). 